\title{
Warum Österreichische Bewegungsempfehlungen?
}

\section{Why Physical Activity Guidelines in Austria?}

\author{
Autoren \\ Sylvia Titze ${ }^{1}$, Thomas Ernst Dorner ${ }^{2}, 3$, Klaus Ropin ${ }^{4}$, \\ Christian Halbwachs ${ }^{5}$, Verena Zeuschner ${ }^{4}$, Thomas Stickler ${ }^{6}$ \\ Institute \\ 1 Institut für Sportwissenschaft, Universität Graz, Graz, Austria \\ 2 Abteilung für Sozial- und Präventivmedizin, Zentrum für Public \\ Health Medizinische Universität Wien, Kinderspitalgasse 15/I, \\ Austria \\ 3 Sozialversicherung öffentlich Bediensteter, Eisenbahnen und \\ Bergbau, Wien, Austria \\ 4 Geschäftsbereich Fonds Gesundes Österreich, Gesundheit \\ Österreich $\mathrm{GmbH}$, Wien, Austria \\ 5 Bundes-Sport GmbH, Wien, Austria \\ 6 Bundesministerium für Kunst, Kultur, öffentlichen Dienst und \\ Sport, Wien, Austria
}

Bibliografie

Gesundheitswesen 2020; 82 (Suppl. 3): S168-S169

DOI 10.1055/a-1189-3424

ISSN $\quad 0949-7013$

(c) 2020. Thieme. All rights reserved.

Georg Thieme Verlag KG, Rüdigerstraße 14,

70469 Stuttgart, Germany

\section{Korrespondenzadresse}

Univ.-Prof. Mag. Dr. Sylvia Titze, MPH

Institut für Sportwissenschaft

Universität Graz

Mozartgasse 14

8010 Graz

Austria

sylvia.titze@uni-graz.at
Die Bedeutung regelmäßiger körperlicher Aktivität für die Gesundheit ist vielfach belegt. Die Weltgesundheitsorganisation (WHO) und die Europäische Kommission verweisen regelmäßig auf den hohen Stellenwert von Bewegung für die Gesundheit und fordern dazu auf, der Bewegungsförderung auf nationaler Ebene mehr Aufmerksamkeit zu schenken. In Österreich wurden Bewegung und Sport als wichtiger Bestandteil nationaler Public-Health-Maßnahmen erkannt - „Gesunde und sichere Bewegung im Alltag fördern“ ist eines von 10 Gesundheitszielen - und es wurden wesentliche Voraussetzungen für die Umsetzung geschaffen [1]. Die Veröffentlichung der ersten Österreichischen Empfehlungen für gesundheitswirksame Bewegung 2010 bildete nicht nur die Grundlage für die Entwicklung eines Nationalen Aktionsplans Bewegung (NAP.b) [2], sondern führte auch zur Entwicklung eines österreichischen Bewegungsmonitorings, dessen Ergebnisse 2017 veröffentlicht wurden [3]. Eine weitere wichtige Rolle spielten die Bewegungsempfehlungen für die Erstellung von Wirkungszielen im Rahmen des bereits oben genannten österreichischen Gesundheitsziels 8 "Gesunde und sichere Bewegung im Alltag durch die entsprechende Gestaltung der Lebenswelten fördern“.

In den zehn Jahren seit der Publikation der ersten Österreichischen Bewegungsempfehlungen [4] waren in vielen Bereichen Weiterentwicklungen zu beobachten, die eine Aktualisierung der Empfehlungen nahelegen. Dazu gehören neben den neuen österreichischen Daten zum Bewegungsverhalten insbesondere die Amerikanischen Bewegungsempfehlungen 2018 [5], die auf einer umfangreichen wissenschaftlichen Dokumentation beruhen. Die gemeinsame Forschungsstelle der Europäischen Kommission hat im Mai 2019 unter dem Titel „Körperliche Aktivität und sitzende Lebensweise“ ebenfalls ein Leitdokument veröffentlicht, in dem der aktuelle Forschungsstand zum Thema zusammengefasst ist [6]. Auf Ebene politischer Zielsetzungen wurden mit dem „Global
Action Plan on Physical Activity 2018-2030“ der WHO Bewegungsförderungsmaßnahmen schließlich vier Aktionsfeldern zugeordnet und 20 politische Umsetzungsstrategien vorgeschlagen [7].

Diese Entwicklungen galt es im Rahmen einer Aktualisierung der Österreichischen Bewegungsempfehlungen zu integrieren. Das Kernelement dieser Empfehlungen bleibt jedoch die Beantwortung der Frage: „Wie viel Bewegung ist empfehlenswert?“ Eine Antwort für verschiedene Zielgruppen zu finden, ist nicht einfach. Zu unterschiedlich sind die Zugänge, weil gute Gesundheitsförderung (und auch Bewegungsförderung) voraussetzt, Bevölkerungsgruppen dort abzuholen, wo sie sich befinden, und Ziele vorzugeben, die erreichbar sind. Diese Ziele sehen für 25-jährige Freizeitsportlerinnen und -sportler anders aus als bspw. für 50-jährige, körperlich inaktive Personen mit Risikofaktoren. Die Empfehlungen für gesundheitswirksame Bewegung sind daher nach Altersgruppen gegliedert. Darüber hinaus wurden in diese Aktualisierung auch spezifische Empfehlungen für Frauen während bzw. nach der Schwangerschaft sowie Empfehlungen für Erwachsene mit chronischen Erkrankungen aufgenommen. Zudem wird explizit darauf hingewiesen, dass die Bewegungsempfehlungen für Menschen ohne und mit Körper-, Sinnes- oder Mentalbehinderung gelten. Es versteht sich von selbst, dass die Altersgrenzen nicht starr sind und bei der Anwendung der Empfehlungen der gesundheitliche Gesamtzustand individuell berücksichtigt werden muss.

In dieser Sonderausgabe der Zeitschrift „Das Gesundheitswesen“ werden die österreichischen Bewegungsempfehlungen samt Hintergrundinformationen dargestellt. Die Kernempfehlungen werden für Frauen während der Schwangerschaft und danach, Kinder im Kindergartenalter und Kinder und Jugendliche [8] sowie für Erwachsene und ältere Erwachsene ohne und mit Körper, Sinnesoder Mentalbehinderung und für Erwachsene mit chronischen Erkrankungen [9] dargestellt. Um diese Kernempfehlungen besser 
interpretieren zu können, werden Grundbegriffe der Bewegungsund Trainingslehre erklärt, der Zusammenhang zwischen Bewegung und Gesundheit dargestellt, und, damit Bewegung keine nachteiligen Folgen für die Gesundheit hat, Informationen in Bezug auf Sicherheit während körperlicher Aktivität gegeben [10]. Das Bewegungsverhalten der Österreicherinnen und Österreicher, Kosten aufgrund mangelnder Bewegung und Umsetzungsempfehlungen auf unterschiedlichen Ebenen zur Bewegungsförderung werden in einem eigenen Artikel dargestellt [11]. Zusätzlich wird in einem Originalbeitrag der Zusammenhang zwischen Bewegungsdeterminanten, dem Bewegungsverhalten und der Gesundheit in den österreichischen Bundesländern aufgearbeitet [12].

Die Mitglieder der Kompetenzgruppe Körperliche Aktivität/Bewegung/Sport der Österreichischen Gesellschaft für Public Health, die als Arbeitsgruppe die österreichischen Bewegungsempfehlungen formulierten, waren von der Überzeugung getragen, dass diese Empfehlungen einen wichtigen Eckpfeiler für gesundheitsorientierte Bewegungsförderung darstellen. Dies setzt jedoch voraus, dass diese Empfehlungen breite Akzeptanz bei all jenen Personen finden, die sich im Sinne von „Health in all policies“ für „Bewegung in all policies “ einsetzen und zu deren Verbreitung beitragen. Daher wurde bei den entsprechenden Zielgruppen Feedback zu den Bewegungsempfehlungen eingeholt und eingearbeitet.

Die Veröffentlichung dieser Empfehlungen ist mit dem dringenden Wunsch verbunden, dass Personen aus dem Bereich Gesundheits- und Bewegungsförderung diese aufgreifen und im eigenen Umfeld einsetzen. Das wäre einerseits ein wichtiger Beitrag dazu, die Bewegungskompetenz und damit die Gesundheitskompetenz der Bevölkerung zu steigern, und andererseits eine Chance, dass Österreich bewegungsfreundlicher wird und mehr Menschen die Vorteile gesundheitswirksamer Bewegung für sich entdecken können.

\section{Interessenkonflikt}

Die Autorinnen/Autoren geben an, dass kein Interessenkonflikt besteht.

\section{Literatur}

[1] Bundesministerium für Gesundheit und Frauen, Hrsg. Gesundheitsziele Österreich. Richtungsweisende Vorschläge für ein gesünderes Österreich - Kurzfassung. Wien: 2. Auflage 2017

[2] Angel B, Chahrour M, Halbwachs C et al. Nationaler Aktionsplan Bewegung NAP.b. Bundesministerium für Landesverteidigung und Sport \& Bundesministerium für Gesundheit, Hrsg. Wien: 2013

[3] BMLV (Bundesministerium für Landesverteidigung und Sport). Bewegungs-Monitoring Bevölkerungsbefragung 2017. Abgerufen am 27. November 2019, von https://www.bmkoes.gv.at/sport/breitensport/breiten-gesundheitssport/Bewegungsmonitoring.html

[4] Titze S, Ring-Dimitriou S, Schober PH et al. \& Arbeitsgruppe Körperliche Aktivität/Bewegung/Sport der Österreichischen Gesellschaft für Public Health Österreichische Empfehlungen für gesundheitswirksame Bewegung: Band Nr. 8 aus der Reihe Wissen (GÖG/FGÖ., Hrsg). Wien: 2012

[5] US Department of Health and Human Services. Physical Activity Guidelines for Americans. 2nd ed. Washington, D.C: US Department of Health and Human Services; 2018. Abgerufen am 27. November 2019, von https://health.gov/paguidelines/second-edition/pdf/ Physical_Activity_Guidelines_2nd_edition.pdf

[6] European Commission Science Hub. Physical activity and sedentary behaviour. 2020; Abgerufen am 27. April 2020, von https://ec.europa. eu/jrc/en/health-knowledge-gateway/promotion-prevention/ physical-activity.

[7] World Health Organization. Global action plan on physical activity 2018-2030: more active people for a healthier world. Geneva: WHO; 2018

[8] Ring-Dimitriou S, Winsperger K, Kayer B et al. Österreichische Bewegungsempfehlungen für Frauen während und nach der Schwangerschaft, Kinder im Kindergartenalter und Kinder und Jugendliche. Das Gesundheitswesen 2020; 82 (suppl. 3): S177-S183

[9] Titze S, Lackinger C, Fessl C et al. Österreichische Bewegungsempfehlungen für Erwachsene und ältere Erwachsene ohne und mit Körper, Sinnes- oder Körperbehinderung sowie für Menschen mit chronischen Erkrankungen. Das Gesundheitswesen 2020; 82 (suppl. 3): S170-S176

[10] Miko HC, Zillmann N, Ring-Dimitriou $S$ et al. Auswirkungen von Bewegung auf die Gesundheit. Das Gesundheitswesen 2020; 82 (suppl. 3): S184-S195

[11] Mayer S, Felder-Puig R, Gollner E et al. Bewegungsverhalten, Kosten mangelnder körperlicher Aktivität und Bewegungsförderung in Österreich. Das Gesundheitswesen 2020; 82 (suppl. 3): S196-S206

[12] Dorner TE, Haider $S$, Lackinger $C$ et al. Bewegungsdeterminanten, Erfüllung der Empfehlungen für ausdauerorientierte Bewegung und Gesundheit: Ergebnisse einer Korrelationsstudie aus österreichischen Bundesländern. Das Gesundheitswesen 2020; 82 (suppl. 3): S206-S216 